\title{
Agreement Technologies Applied to Transmission Towers Maintenance
}

\author{
Pablo Chamoso ${ }^{a,}$, Juan F. De Paz ${ }^{a}$, Fernando De La Prieta ${ }^{a}$, and Javier Bajo Pérez ${ }^{b}$ \\ ${ }^{a}$ Department of Computer Science and Automation Control, University of Salamanca. \\ Plaza de la Merced s/n 37008, Salamanca, Spain. E-mail: \{chamoso, fcofds, fer $\}$ @ usal.es \\ ${ }^{b}$ Faculty of Informatics, Department of Artificial Intelligence, Technical University of Madrid. Campus \\ Montegancedo, Boadilla del Monte, 28660 Madrid, Spain.
}

E-mail: jbajo@fi.upm.es

\begin{abstract}
Among the most indispensable elements required by a city is electric power and the transmission towers used for its distribution. These towers have underground electrodes which must be reviewed on a regular basis by controlling different parameters to ensure that electrical resistance is in a secure range in order to avoid problems and risks. Using artificial intelligence, it is possible to ensure proper maintenance of the towers by estimating the required values and proposing a re-duction in the size of the population sample, which will min-imize the cost of operation. The use of an intelligentagent virtual-organization based architecture is proposed within this working environment. By using mathemati dl estimation models and agreement based negotiations, $t$ architecture is capable of maximizing estir tir as na minimizing associ-ated costs. The proposed rode as bec $_{\text {. }}$ evaluated with the developed software throu' a re.' case study, which permit-ted us to validate the rrposed approach.
\end{abstract}

Keywords: transmission towers, agı ment technologies, CBR system, agents

\section{Introduction}

One of the main areas of Information Technology (IT) focuses on the application of emerging techniques and technologies in different everyday objects. The aim is to interconnect these objects and provide them with the ability to acquire some degree of knowledge and/or intelligence, which makes it possible to obtain new benefits and features. This new paradigm is known as the Internet of Things (IoT). One of the main fields of research and application of IoT are cities. Using IoT techniques can lead to the creation of Smart Cities, which are associated with the pursuit of benefits for citizens. These benefits may affect the society directly by offering new or improved services;

\footnotetext{
*Corresponding author
}

or indirectly, by usı $\sigma$ th 2 application of IT to achieve savings.

At present th ten incy is to transform a portion of a city's ssets no intelligent entities, interconnecting them by sin large-scale networks to provide data prar *ally automatically and instantaneously. Howev $r$ are different assets in a city that are not suitable for his transformation to IoT, either because of their nature or the cost entailed by adapting the exist$\mathrm{g}$ infrastructure. One of these assets is the focus of this article: the Transmission Towers (TTs) that transport electricity. Many are located in isolated points, where even communication through mobile technologies is limited and the cost of the required equipment to monitor and control them is too high to be included or placed on every TT. However, it is important for the TTs to benefit from smart city features, which will undoubtedly result in an economic benefit.

The main benefit of IoT, in this case, is the reduction of maintenance costs, which in this type of infrastructure is complex because these costs are necessary to guarantee periodic revisions in each TT, which include measuring different parameters to ensure the security basics of the installation. In addition, such revisions are imposed by law in most developed countries, although the specific processes to be followed are defined in each individual country. The threshold value of the observable parameters in each revision is also defined, which guarantees the safety of the electric line. Undoubtedly, having to revise all TTs represents a high cost, mainly due to the great distance they cover, their inaccessibility and the need for specialized equipment and personnel. However, this cost can be reduced if the number of TTs to check is minimized. Obviously, there must be a high level of confidence that the TTs that are not reviewed are not going to fail. 
Therefore, the problem consists of identifying the TTs that should be physically checked. The complexity is determined by the large amount of TTs. In fact, only in Spain there are over 42,000 kilometers of high voltage power lines [6], many of them, supported by more than 600,000 TTs. The solution is approached from a perspective of Artificial Intelligence (AI), through the use of Virtual Organizations (VOs) of intelligent agents. These autonomous entities use distributed decision making processes and incomplete information, features that cater to the proposed problem. Agents in VOs create stratified sampling to analyze the state of the lines, and the samples are then used to analyze the condition of a similar tower over ground with similar resistivity. Taking this into consideration, the system will determine the number of TTs requiring review. Agents will then have to cooperate, negotiating with each other in order to determine the final sample of TTs to be reviewed, which will be those with the highest impact in the whole set of selected TTs. This will allow the system to guarantee high confidence levels for the TTs that were not reviewed. To this end, we propose a framework for negotiations based on Agreement Technologies (AT), which provides the organizational system with the capability of finding and learning sol :tions when the problem to solve involves reaching a agreement among the agents, with autonon and nteractions between stakeholders being th na. keys. The agents can use neural networks to pre "ct rusistance depending on several parameters. 1 proposed model is evaluated in a simulation env ${ }^{+}$nm nt, which, by using real data from TTs in to validate the results of the sa ples and predictions obtained.

The following section presents the problem in greater detail, as well as existing related works. In section 3 the proposed multi-agent system is detailed, followed by the model of argumentation in section 4 . The evaluation of the system is presented in section 5. Finally, section 6 presents the detailed conclusions and the future work.

\section{Problem Description}

A TT is a structure, usually made of steel, acting as a support for aerial electric conductors which are used to transport electrical energy. Each TT has (i) an associated configuration set, defining the model of the structure and the underground electrodes, the location, and other static aspects; a (ii) state, which will group a set of observable magnitudes that vary over time; and a (iii) revision history, which stores the evolution of data (both static and dynamic). TTs are also characterized by a parameter that defines if they are frequented (in a city or an accessible place) or not.

One of the main drawbacks and the main reason that TTs must be regularly reviewed is their exposure to people, who can walk around them or even touch them. So frequented TTs are more dangerous than not frequented ones and law usually defines different criteria to review them. A malfunction can cause that person to suffer serious or fatal ei ctrical shock, in addition to causing other problems vith energy distribution. In order to guarante - that sin tions like this never happen, and for addit: al se urity easons, the regulations of each cour ${ }^{2}$ fo $n$. revision of the elements involved in the ( strib ion f electricity through high voltage pow in (i the case of Spain, the legislation is publ shed 15]). The revision of a TT involves a high cost $w_{\text {. }}$ laving to manage the displacement of tec' AItcal equipment and specialized machinery to each T1 Jurtr ermore, the process requires previous preparatior, once the towers are active high voltage lines. By reducing the number of supports to be measured, 1.e value and time of completion of the operation is definitively decreased.

Most of the problems that can arise in a TT depend on their earth leakage. To achieve a good earth leakage, each TT has a number of buried or partially buried electrodes. These electrodes are conductors that remain in contact with the ground to (i) assure the grounding of static charges or atmospheric electrical discharges; (ii) limit the flow and contact voltages in the vicinity of the support; or (iii) limit the unintentional contact voltage with higher voltage systems. Flow and contact tensions are two magnitudes with complex measurements, but they are related to the grounding voltage. Therefore, the electrodes must be properly maintained to ensure they have a resistance that is preferably low, offering sufficient capacity for current conduction.

In general, a material resistivity $(\rho)$ is defined as the ratio of the magnitudes of the electric field and current density, given that a perfect conductor would have a resistivity equal to zero, while a perfect insulator would have infinite resistivity. Based on this value, it is possible to determine the ability of a conductor to act as grounded electrodes; that is, its ability to derive the current that can flow from the TT.

Soil resistivity in particular depends on the materials used in the floor where the TT is located, rela- 
tive humidity and ambient temperature. The TT should not exceed a maximum value of resistance of $20 \Omega$, although it may vary according to the soil resistivity. It must be clear that flow and contact tensions are two magnitudes with complex measurements compared to the grounding resistance, and that there is a relationship among the three of them; therefore, the parameter with the most essential measurement is the grounding resistance.

Wenner method [17] is used to measure resistivity, which defines the soil resistivity as:

$$
\rho=\frac{\text { Resistance }}{K_{R}}
$$

Where $\rho$ is the soil resistivity, Resistance is the TT resistance and $K_{R}$ is the coefficient of resistivity.

This paper attempts to speed up the measurement task by estimating the most appropriate TTs and designing a sample of different lines in order to validate the state of the towers. To do so, it is necessary to begin with information (locations) on a set of TTs in Spain, allowing us to know (i) the type of terrain over which it has been raised, including its (approximate) resistivity, and the distance to the rest of towers (ii) the type of each tower, which in turn has its own coefficient of 1 :sistivity, and (iii) the line they belong to, which is in portant because ideally each line consists o, 1 e sa. e type of towers; although this may not be $\iota$ ca with older facilities. With this information, se nple are carried out to validate the state of the towe and new configurations of towers are $d$ cigned.

\section{Related wo}

The problem of maintenance on power lines is required mainly because of security reasons. There are different types of maintenance [2], which are presented below. First, (i) corrective maintenance consists of fixing existing bugs for the system to start working correctly again. This type of maintenance can be divided, according to its required planning, into planned or unplanned maintenance. The planned corrective maintenance is a technique that ensures a reduction in costs and duration of the repair. For this reason, classification algorithms [11] or neural networks [15] [16] have been applied to address problems of ice accumulation [18] [10] as well as the prediction of physical deterioration of machinery (generators and transformers) [20] [12]. Next, (ii) preventive maintenance consists of reducing equipment failures by seeking solutions to problems before they happen. During the process, the service may be interrupted to carry out conservation work, which must be planned [1] [7]. The (iii) predictive maintenance arises as a complement to preventive and corrective maintenance. It consists of monitoring a number of parameters for further analysis, looking for possible anomalies. Finally, (iv) proactive maintenance is a preventive maintenance strategy used to stabilize the reliability of the machinery or equipment. Within this maintenance, the work proposed in [4] stands out, where the authors manage to theoretically model the impact of proactive maintenance work. Later, the use of the concepts of residual use ul life and the models of each phase of failure male it 1 sssible to provide optimal planning from an nomm soint of view.

Although previous ork $h \mathrm{~s}$ bee $\mathrm{l}$ done in the maintenance of TTs, th - ar no known jobs trying to predict the magnit des $t_{1}$ at $g$ arantee the safety of the line. This pi nel o v ork makes it possible to predict and sample the 1 imber of TTs to revise through the use of intellı_. agents. The system tries to predict the state of the lines by sampling the towers according sev ral parameters such as ground resistivity and type c..ctrode, and other parameters such as the last revision and information taken from other towers. This $\checkmark$ ill determine a subset of towers to review, which will reduce maintenance costs.

In addition, and in order to determine the best subset of towers to be reviewed, this work uses AT. Recent developments in argumentation-based agreement models such as [3] or [13] have provided the necessary technology to allow agents to engage in argumentation dialogues, harmonize beliefs, negotiate, collaboratively solve problems, etc. Consequently, the ability to determine which TTs have to be reviewed is the main problem to solve once the number of TTs to review is known. To do this, the use of AT has been included, since its power of argumentation is based on the use of a CBR (Case Based Reasoning), thus making it capable of learning as it is used. The agents design the stratum according to specific parameters, and the final towers are selected through a negotiation process that determines the TTs whose revision have more influence in the remaining towers which were not reviewed.

\section{Multi-Agent System Architecture}

Having clearly identified the problem to be addressed, namely, the reduction of operational costs in the maintenance of TTs, we now posit a solution that 
uses an innovative approach by which the set of TTs to review is determined through statistical sampling. Statistical samplings are used to estimate which resistance values are closest to a value with a maximum error and with known confidence levels, which makes it possible to avoid performing a complete analysis of a line with TTs having similar features.

The problem can be addressed by defining specific types of VOs, which use information gathered during the inspections and review electrical lines. Using this information, it is possible to (i) predict the status of each TT and (ii) determine what TTs should be measured when companies have to make revisions in an area or line.

To create the model of interaction it is first necessary to analyze the motivation for potential users of the system. Externally, we have identified two interest groups through an analysis of requirements. First is the user, or client, who uses the application to manage the tasks related to the power lines and their maintenance. Second is the provider, who is dedicated to updating the system information, adding new data as inspections or reviews are conducted. The VOs can be framed in a dynamic but simple environment, since the output will always have the same format and meaning even thou $h$ new elements appear in the system.

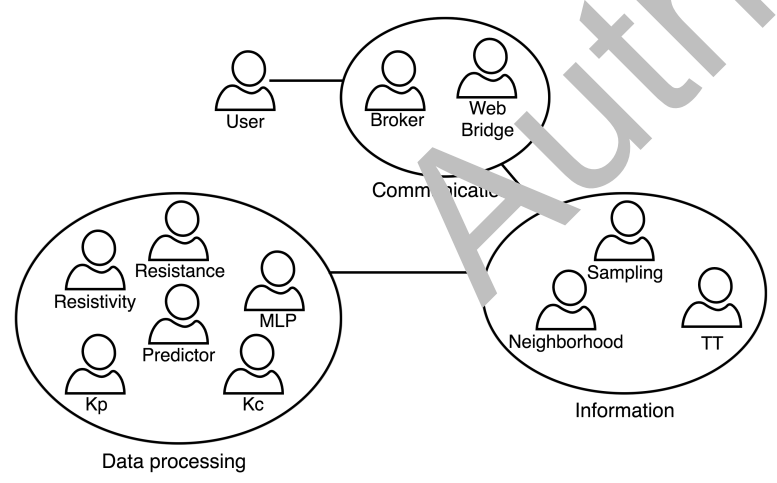

Fig. 1. Defined VOs schema.

Using this initial analysis of roles and external environment, we designed three VOs as part of this work. They can be seen in Figure 1 and are listed below:

- Communications VO. This VO encapsulates the functionality that allows the final user to communicate with the rest of the system.

- Information VO. This VO encapsulates the agents whose functionality is related to basic information management and storage.
- Data processing VO. This VO encapsulates the agents in charge of processing all the information in advance, and provides different AI abilities.

These VOs contain the following roles:

- User. Represents the potential user of the system who will use the prediction tool to achieve optimal maintenance of power lines. It has access to the entire information repository. It is also responsible for starting a prediction process for a set of high voltage power lines. Finally, it also represents external entities thi perform actual measurements in the TTs. 1. vstem provides reliable information

- Web bridge. This , le is mplemented by an agent in charge $c$ the ntc mation exchange between the systam nd a user. It receives REST or socke reque ts which are transformed into FIPAACL a. ' vi e versa.

- roker. This role receives petitions from the Web 10 e agent and forwards them to the adequate orat.

- TT. Represents every TT. This role is in charge of storing all individual states. Therefore, it contains information on the configuration, status, position, revision histories, etc.

- Sampling. Retrieves the information of the selected towers and designs a sample over them. The agent retrieves the towers of the selected lines and designs a stratified sampling in order to reduce the number of tower reviews to carry out and perform a statistical analysis over the whole line.

- Neighborhood. Responsible for neighbor discovery of the tower agents. Able to access its information and exchange it with those agents who can know about it.

- Predictor. This role is implemented by an agent that estimates the state of different parameters associated to a TT in the system. This agent must have access to the repository revision histories and information of the TT to incorporate extra information on the estimation.

- Resistance. It is in charge of predicting the TTs resistance value according to the method described in the "Proposed System" section.

- Resistivity. It is in charge of predicting the ground resistivity value according to the method described in the section "Proposed System".

- MLP. Incorporates a neural network model to predict different requested values. 
- Kp. This agent uses the MLP agent to predict the step potential $(K p)$ values.

- Kc. This agent uses the MLP agent to predict the touch potential $(K c)$ values.

The next section describes the interactions between these agents through the model of argumentation, detailing the way the agents autonomously agree on the revisions.

\section{Proposed System}

The proposed negotiation model is presented throughout this section. In the first subsection, a required previous step is explained; in the next subsection, a general overview of the model is also presented; then the various designed argumentation mechanisms are presented; and finally the negotiator agent architecture is presented.

\section{Initial Step}

The revision process starts when the user requests to review a set of TTs by using the software. The syster then performs a series of actions. First, all the res :tance values for every TT are estimated an ${ }^{1}$ th $P$ 'IS Percentage $(T P)$ is updated. The $T P$ val e $\mathrm{rc}_{\text {, resen. }}$ the reliability on the estimation and it depu. "s dn ctly on the number of TTs with similar chara eris that have been considered for the estimat $n$.

To carry out the prediction: on he res. nnces of the TTs, the system incorporates , e B - reasoning cycle as shown in Figure 2. The c ses memory includes information about the TTs and nearal network models that have been trained previously, and makes it possible to carry out the prediction. TTs are grouped by the $K_{r}$ value, which means they are grouped by their technical characteristics and every group has an associated trained neural network. Every case is defined with (2).

$$
C^{k}=\left(R, \rho, K_{r}, h, t\right)
$$

Where $C^{k}$ is the case group $k, R$ is the resistance, $\rho$ is the ground resistivity, $h$ is the ground humidity, and $t$ is the temperature.

When a new TT is received to be reviewed, the CBR reasoning cycle starts. The first stage is "Retrieve", where all the related cases are retrieved by the system. Those related cases belong to the similar theoretical $K_{r}$ group. The theoretical $K_{r}$ groups are predefined according to the type of TT (we have three groups in

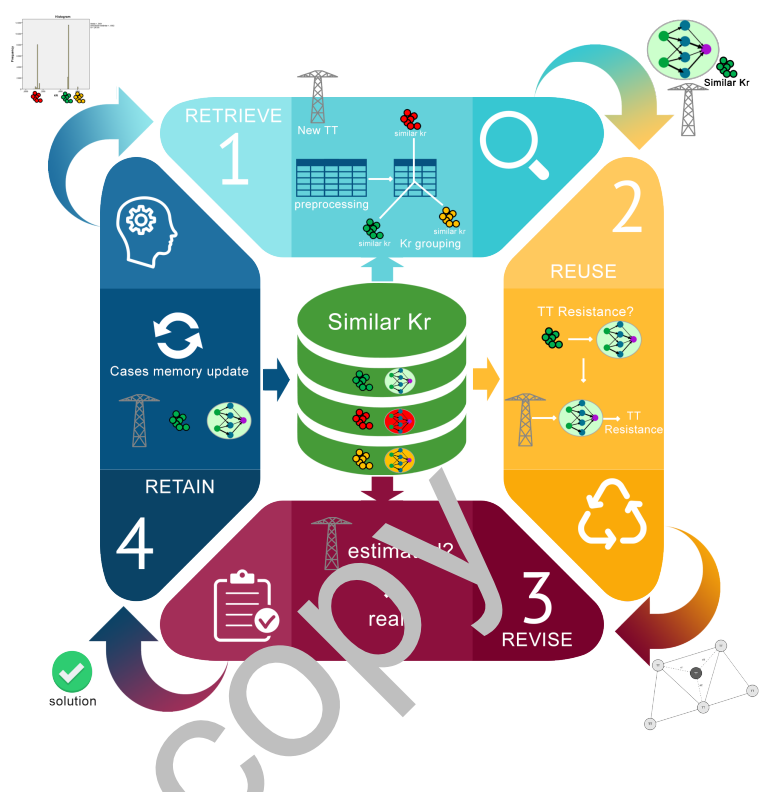

Fig. 2. CBR olc or the resistivity and resistance estimation.

the av lin le of Spain). If the neural network associated to the r oup is already trained, it is also retrieved by the system. Otherwise, the neural network associated the group is trained in the next stage, called reuse.

Once the system has a neural network trained at the reuse stage, the system proceeds with the resistance estimation. The estimation is performed through the neural network, but it is necessary to know or to estimate the value of the ground resistivity. If the ground resistivity has to be estimated, $\bar{\rho}$, it is calculated with the equation (3) which is the weighted average where closest neighbors have more influence in the result. A weighted deviation, $\sigma$ is also calculated with the equation (4) to provide the possible error of the calculated ground resistivity value. A visual example is shown in Figure 3.

$$
\bar{\rho}=\sum_{i=1}^{n} \rho\left(T T_{i}\right) * \frac{D_{\max } / d_{i}}{\sum_{j=1}^{n}\left(D_{\max } / d_{j}\right)}
$$

Where $\rho\left(T T_{i}\right)$ is the ground resistivity for the ith TT, $D_{\text {max }}$ is the longest distance, $d_{i}$ is the distance between the ith TT and the point of the current or new TT.

$$
\sigma=\sqrt{\frac{1}{n} \sum_{i=1}^{n}\left(\rho_{i}-\bar{\rho}\right)^{2} \frac{D_{\max } / d i}{\sum_{j=1}^{n}\left(D_{\max } / d_{j}\right)}}
$$

The multilayer perceptron (MLP) networks are defined according to this structure: four inputs $\left(\rho, K_{r}, h, t\right)$, 9 neurons in the hidden layer, 1 output with the resistance. The activation functions are sigmoid. 


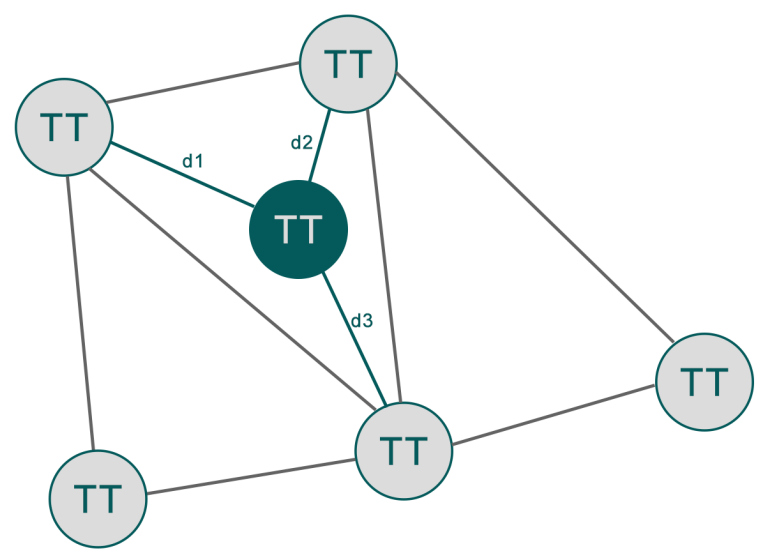

Fig. 3. Resistivity estimation example.

If the TT is revised by the maintenance company, real values are included in the system by using the developed software (revise stage). In these cases, the $T P$ value are updated to 100 .

Finally, if measured, values are inserted in the database and the related neural network is retrained when the number of new cases reaches a predefined threshold (retain stage).

\section{Sampling Algorithm}

As explained before, one of the objec res ${ }^{f}$ this work is to develop a stratified sampling $>c_{a}-v$ out a statistical analysis of the lines. The amplc are stratified according to the $K_{r}$ and groun ${ }^{1} r$ sistivity in order to analyze similar towers for an stratum. Once the stratified sampling has bee finished, the TTs to be reviewed will be selected by using a negotiation process. Ground resistivity should not change considerably, because it depends to a greater degree on the composition of the terrain. The size of the sample is calculated for each stratum, and is defined to calculate the average resistance with a level of confidence and error. The population is divided into three groups according to the kind of electrodes and the associated $K_{r}$. Additionally, the system calculates the deciles for each group of three. The size of the sample is then calculated for every decile. The equation (5) defines the size of the sample, the error value is defined according to equation (6).

$$
\begin{aligned}
& n=\frac{z^{2} \sigma_{i}^{2} N_{i}}{e_{i}^{2}\left(N_{i}-1\right)+z^{2} \sigma_{i}^{2}} \\
& e=\operatorname{Max}\left\{\left(d_{i+1}+d_{i}\right) / 2 \cdot k,\left(\left(d_{i+1}+d_{i}\right)-\rho_{i}\right) \cdot k\right\}(6)
\end{aligned}
$$

Where $z=1.96, \sigma$ is the variance, $N$ is the population size, $k$ is a constant defined by $0.15, \rho_{i}$ is the lower value of the resistivity in the decile $\left.i, \rho_{(} i+1\right)$ is the upper value of the resistivity in the decile $i$.

For each decile we have to select the $n_{i}$ elements as described in Algorithm 1. Once finished, the negotiation process starts.

\section{Input: GroupsKr Output: $n$}

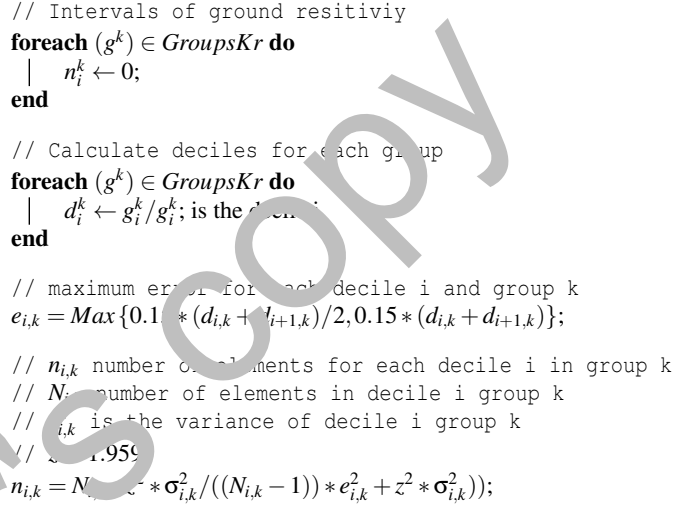

Igorithm 1. Sampling

\section{Negotiation Description}

One of the most important parts of the system is the negotiation between agents, whose objective is to determine the TTs to be reviewed after the number of TTs to reviewed has been calculated. The negotiation begins the moment the user agent requires the system to measure the sample of the TTs within a region, the values are estimated by the system (after the reuse stage), and the sampling algorithm has been executed. At that time, each TT within the territory is associated with a tower agent which checks its current $T P$. If $T P$ equals 100 , it means that the state of its parameters is reliable and does not require review, and therefore the agent does not participate in the negotiation. Otherwise, it will participate by exchanging the arguments with its peers until a valid proposal is found.

During the negotiation process, all agents are connected and collaborate in pursuit of a common goal, which is to achieve the best solution based on their experiences. However, agents may have opposite interests:

- Safe: agents that promote this value will select those solutions that increase their $T P$. 
- Economic: agents that promote this value will select those solutions involving the lowest revisions.

- Neutral: these agents seek to maximize their TP and reduce costs as much as possible, with a more relaxed posture than the others.

Each agent has a type of individual proposal (safe, neutral or economic) for the TT they represent.

Initially, to argue the individual proposal, it is necessary to obtain the $T P$ from the Predictor agent for the worst outcome from not revising: Worst $T P(W T P)$. Once this value is known, the Tower Agent determines its role in the negotiation, evaluating its history and checking two situations: i) if the tower previously had a $T P$ lower than the $W T P$ (Previous lower $T P, P T P$ ); and ii) consulting the Neighborhood Agent to know the $T P$ of its neighbors (NTP).

If there has not been a situation where $P T P<W T P$, the position that the Tower Agent will adopt will be the safe option.

On the other hand, if it is true that $W T P>\frac{\sum_{i=1}^{n} N T P_{i}}{n}$, where $n$ is the number of neighbors, it will adopt an economic position, prioritizing the revision of its neighbors.

Otherwise, its position will be neutral.

Thus, different situations may occur during the $n$. gotiation:

(a) Agents involved accept the proposal be ause hey coincide, so the TT represented is a dea to the sample to be reviewed.

(b) Agents involved do not a cep. 'acause more than one wishes the TT they rep "sent to be revised.

(c) Agents involved do not a cept because none wants the TT represented to be revised.

(d) The agents involved have a neutral perspective.

In cases $b$, $c$ and $d$, the agents with the safe solution must negotiate to determine which TTs are finally reviewed; an exchange of arguments supporting each position will use a CBR model, as detailed in subsequent sections.

\section{Negotiation Mechanism}

As previously noted, when defining a model of negotiation based on agreements in which arguments are used, it is first necessary to determine a number of mechanisms that support the negotiation process itself. The most important mechanisms are communication language and domain language.
To begin, the FIPA ACL (Foundation for Intelligent Physical Agents' Agent Communication Language) [8] is selected as the language of communication primarily because of its semantic capacity, as it includes locutions to express acceptance, rejection, proposal applications, requests, inquiries, statements, declarations, etc. Communication was made through the use of PANGEA [19] [14], which allows for a cross-platform distributed development and disengages the specific functionality of the application of basic functions, such as access to data or norms of communication between as nts. For this negotiation, 4 types of locution on FIP - ACL are to be used: (i) inform: desire_to_reva (LS, desire_not_to_revise (L4), prefer_to_revise L5), refer not_to_revise (L6), withdraw_dialogu $\quad 1$, (i) propose: open_dialogue (L1); agree_to_r vise ( 49 , (iii) accept-proposal: enter_dialogue $(-2)$, or _not_to_revise (L10); (iv) refuse: refuse_to_r vise (. 7), refuse_not_to_revise (L8).

Once the 1. uage of communication is defined, it is cessary to define a domain language, allowing the ssa e of meta-information separately or together with ner locutions. To this end, we must define an ontology compatible with IFAP in order to carry out 1. e decision-making process that will determine which TTs are reviewed. Its class structure is defined in the table 1 .

The structure is composed of two abstract classes (Concept and Predicate). The other classes are defined in the way shown in the diagram. For a better understanding, the type attributes Attributes, Constraint and Valuation, must be defined. To begin, (i) attributes reflects parameters that are associated with the TT and which the Tower Agent already knows. They are needed when estimating the $T P$ of the neighbors in the CBR. In particular, they are the model of the TT $\left(K_{r}\right.$ value), the type of terrain on which the tower stands (determined by the ground resistivity value), the UTM (Universal Transverse Mercator) coordinates where the tower is located, and the number of neighbors (provided by the Neighborhood Agent). The value reflected by (ii) constraints refers to its current $T P$, followed by the $T P$ it would adopt if each of its neighbors were revised. If the safe role was initially taken, it means that its $W T P$ is the smallest one of the values sent and there are no lower values in its history. In the case of playing an economic role, it means that its $W T P$ is larger than the smallest one of the values. Finally, (iii) valuation provides the level of interest of an agent in the review of each of the possibilities (it and its neighbors). In the case of adopting a safe role, the value that is associated 
Table 1

Negotiation ontology.

\begin{tabular}{|c|}
\hline Concept \\
\hline $\begin{array}{l}\text { AgentAction } \\
\text { - Open_dialogue :area (String) } \\
\text { - Agree_to_revise :proposal (Tower instance) } \\
\text { - Revise :proposal (Tower instance) } \\
\text { - Not_revise :proposal (Tower instance) }\end{array}$ \\
\hline AgentID :agent identifier (String) \\
\hline Tower :attributes (String) \\
\hline Revision Requirement :constraints (String) \\
\hline $\begin{array}{l}\text { Revision Requirement Valuation :constraints (String) :valuation } \\
\text { (String) }\end{array}$ \\
\hline Predicate \\
\hline $\begin{array}{l}\text { Desire_to_revise :tower (Tower instance) :revision requirement } \\
\text { (Revision Requirement instance) }\end{array}$ \\
\hline $\begin{array}{l}\text { Desire_not_to_revise :tower (Tower instance) :revision } \\
\text { requirement (Revision Requirement instance) }\end{array}$ \\
\hline $\begin{array}{l}\text { Prefer_to_revise :tower (Tower instance) :revision requirement } \\
\text { validation (Revision Requirement Validation instance) }\end{array}$ \\
\hline $\begin{array}{l}\text { Prefer_not_to_revise :tower (Tower instance) :revision } \\
\text { requirement validation (Revision Requirement Validation } \\
\text { instance) }\end{array}$ \\
\hline Withdraw_dialogue :area (String) \\
\hline
\end{tabular}

to its current $T P$ would take the maximur va' $n(1)$, while the neighbor with the worst $T P$ af $\neg \mathrm{r}$ th revie, would take the minimum (0). If taking an econ mic role, it will not be to be revised, so it $\mathrm{t}^{11} \mathrm{cL}$. Jse to take the minimum value $(0)$ and the 1 . ighb . with the highest $T P$ value will take the nic : mun alue (1).

Dialog model locutions are $s^{\mathrm{r}} \mathrm{swn}$ Delow (sender and receiver fields must be inclu ed in ACL messages but they are not included to facilitate the reading) with real data of two TTs selected from line L363923 to show a real example of the values.

The first TT has the following attributes: the $K_{r}$ value is 0.4991 ( $K_{r}$ group 2 ), the ground resistivity is $25.1248 \Omega \mathrm{m}$, it is placed in $30 \mathrm{~N} 544346.557 \mathrm{mE}$ $4622676.576 \mathrm{mN}$ (UTM) and it has 4 neighbors. Its $T P$ value is 50 and in the case that its neighbors are reviewed, it would have a $T P$ value of 45 for the first one, 30 in case the second neighbor is reviewed, 35 in case of the third one, and 40 in case the fourth neighbor is reviewed.

The second TT has the following attributes: the $K_{r}$ value is 0.4995 ( $K_{r}$ group is 2 ), the ground resistivity is $23.832 \Omega \mathrm{m}$, it is placed in $30 \mathrm{~N} 556817.591 \mathrm{mE}$ $4622764.95 \mathrm{mN}$ (UTM) and it has 2 neighbors. Its $T P$ value is 90 and in the case that its neighbors are re- viewed, it would have a $T P$ value of 85 for the first one, 80 in case the other neighbor is reviewed.

With these values, the following example of locutions could be used in the negotiation:

- L1: open_dialogue(.): propose (AgentAction (Open_dialogue :area “L363923”)).

- L2: enter_dialogue(.): accept-proposal (AgentAction (Open_dialogue :area “L363923”)).

- L3: desire_to_revise(.): inform (Desire_to_revise (Tower :attributes " $0.4991,25.1248,544346.557$, 4622676.576, 30, 4” :constraints “ $50,45,30,35$, 40")).

- L4: desire_not_to_revise 1.1 form

(Desire_not_to_rer \&se ( Tower :attributes “0.4995 ,23.832, 556817. '1, 4t 2276+.95,30,2” :constraints $90,5, \mathrm{~T}, \mathrm{\prime})$ ).

- L5: prefer_ ᄀ_rev ,e(.): inform (Prefer_to_revise (Towe :attrihutes "0.4991, 25.1248, 544346.557, $46226,5.57$ f , 30, 4” :constraints “ $50,45,30,35$, 10 " :valuation “(1 0.800 .40 .6$)$ ”)).

- I' refer_not_to_revise(.): inform (Pr $\mathrm{Pr}$ er_not_to_revise (Tower :attributes “0.4995, 23.832, 556817.591, 4622764.95, 30, 2” :constraints “90, 85, 80" :valuation "( $\left(\begin{array}{lll}0 & 1 & 0.7\end{array}\right)$ ”)).

- L7: refuse_to_revise(.): refuse (AgentAction (Revise (Tower :attributes "0.4991, 25.1248, 544346.557, 4622676.576, 30, 4”))).

- L8: refuse_not_to_revise(.): refuse (AgentAction (Not_revise (Tower :attributes "0.4995, 23.832, 556817.591, 4622764.95, 30, 2”))).

- L9: agree_to_revise(.): propose (AgentAction (Agree_to_revise (Tower :attributes "0.4991, 25.1248, 544346.557, 4622676.576, 30, 4”))).

- L10: agree_not_to_revise(.): accept-propose (AgentAction (Agree_not_to_revise (Tower :attributes " $0.4995,23.832,556817.591,4622764.95$, $30,2 ”))$ ).

- L11: withdraw_dialogue(.): inform (Withdraw_dialogue :area "L363923").

Given the data in this example and the characteristics of the two towers described above, if they had to negotiate, an agreement would be reached quickly. The reason is that the situation (a) described in the subsection "Negotiation Description" would be in effect, so the first tower would be the TT proposed for review instead of the second, because the $T P$ value in the next revision process will be the maximum possible. If the negotiation between them were to take place, the simplest negotiation process would start, being composed of the sequential exchange of locutions 
L1 (open_dialogue(.)), L2 (enter_dialogue(.)), L3 (desire_to_revise(.)) and L9 (agree_to_revise(.)). After the revision process, the first TT would have a $T P$ value equal to 100 and the second TT, 85 (because the first TT was the first neighbor of the second TT).

\section{Negotiator Agent Model}

Having presented a description of the model of negotiation and support mechanisms, this section will now present the structure of the negotiation agents. Figure 4 represents the structure of the Tower agent, an Argumentation-based Negotiator (ABN) which is a fundamental trait. As shown, the agents have the possibility of explicitly exchanging meta-information.

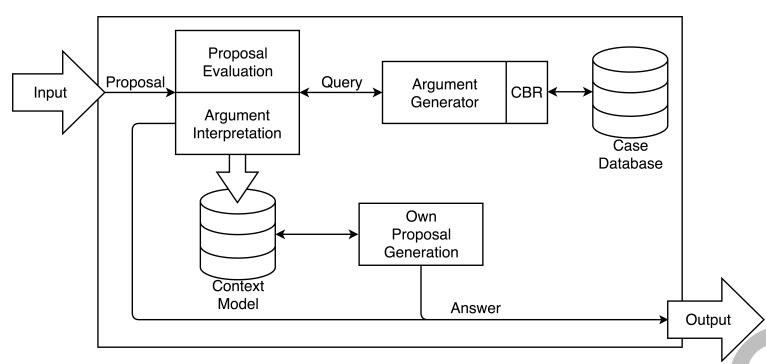

Fig. 4. ABN structure.

The most important elements of the pre, ${ }_{1}$ sec $4 B N$ structure, starting with the context mode wi now be presented, followed by a description f how he system is able to make predictions, a c nally rgumentation model, which is where the CB'. $\mathrm{s}$, tem resides.

\section{Context Moa !}

When establishing the negotiation, the $T P$ value is important, but the arguments must also consist of other parameters such as the type of support, the type of terrain, the position of the tower and the number of neighbors.

Thus, the (i) type of support ensures that $K_{r}$ values vary in the threshold range, guaranteeing that the resistances of the electrodes are similar. Ground resistivity ( $\rho)$ also depends on the soil resistivity, so the terrain is another parameter whose influence is similar to that of $K_{r}$; however, it is a variable parameter that depends on environmental conditions, so it is less influential than the type of support.

The (ii) position of the tower is important because it indicates the distance to each tower. In nearby distances of less than $5 \mathrm{~km}$, and given the same type of terrain, the resistance value of the electrodes should be similar. Each Tower Agent contains information about the position of the support represented by UTM coordinates.

Then, the (iii) number of neighbors parameter influences the negotiations, because the greater the influence over its neighbors, the higher the priority of measuring the tower.

\section{Argumentation Model}

In this work, the argumentation is carried out from a peer-to-peer approach, so that each TT argues why it should be revised, TTs with opposing interests evaluate the argument and accept it or ot according to their own evolution. The evolutio. is validated by the TT that receives the argum ent $\mathrm{c}$ sed $\mathrm{o}$, the case database. The scheme followed bas $\mathrm{d}$ or the argumentation model described $n[s$

Initially, the $i T_{r}$ the wants to be revised sends its argument $\left(\Lambda_{i}\right)$ to tII $T T_{i}$ with which it must negotiate. In the a vum nt the characteristics of the $T T_{r}$ are included and also the variation in the $T P$ of the $T T_{i}$, wl ere $m$ is the current value and $T P_{i}^{\prime}$ is the value that it will $\mathrm{h}$ - $\mathrm{e}$ if it accepts the proposal of $T T_{r}$.

$$
A_{i}=\left(T_{i}\left(K r_{r}, R_{r}, d_{r}, n\right), T P_{i}, T P_{i}^{\prime}\right)
$$

On the basis of this received argument, the $T T_{i}$ searches the cases in the case database in order to evaluate the parameter $T P_{i}^{\prime}$ of the received argument. In case of coincidence, proceed as follows:

- If $T P_{i}<=T P_{i}^{\prime}$ then the contrary position is accepted.

- If $T P_{i}>T P_{i}^{\prime}$, the value of the $T P$ for the opponent $\left(T P_{r}\right)$ in case of reviewing the $T T_{i}$ is predicted $\left(T P_{r}^{\prime}\right)$. If the difference between $T P_{r}$ and $T P_{r}^{\prime}$ is lower than the difference between $T P_{i}$ and $T P_{i}^{\prime}, T T_{i}$ answer by arguing this situation. In case of $T P_{r}-T P_{r}^{\prime}>T P_{i}-T P_{i}^{\prime}, T T_{i}$ would accept the initial proposal.

\section{Results}

As a result, a software to control all the reviews and the estimations is provided (Figure 5). With that software, basic functionality can be used to manage all the information (Create, Read, Update, Delete), and the advanced functionality associated to the cognitive part of the system is also available.

The advanced functionality can be accessed by using two main blocks of the software tool which are marked 


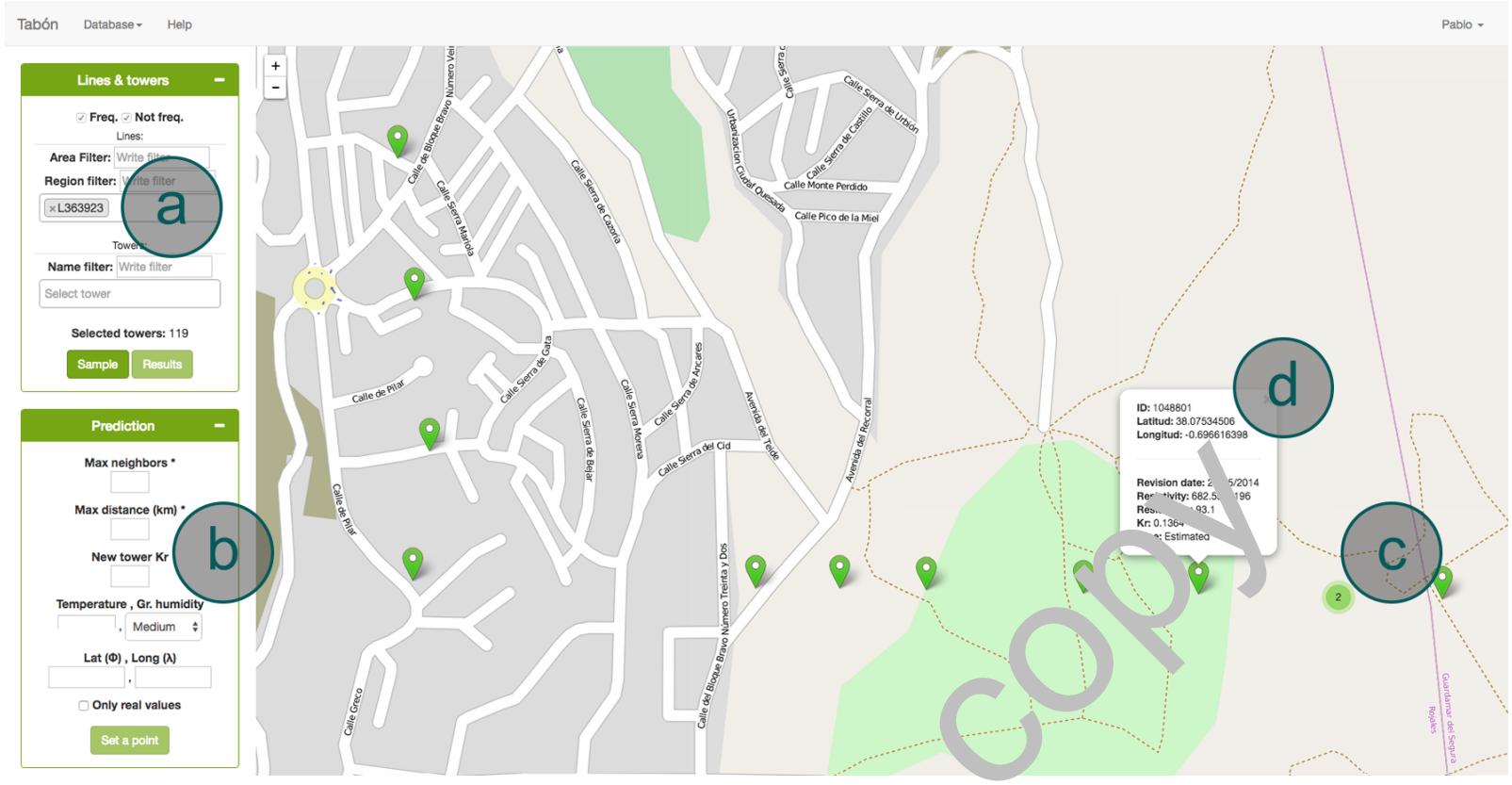

Fig. 5. Softwa int face

in Figure 5 with the letters ' $a$ ' and ' $b$ '. In the ' $a$ ' block, different filters like line name, TT name or TT typ (frequented or not frequented) can be applied to sel $\mathrm{tt}$ electric power lines (sets of TTs) or individual T ${ }^{\mathrm{T}}$ r $^{\mathrm{rol}}$. the database (which can be managed in the ' $L$ tabas, option, in the header menu). Once the se $f \mathrm{~T}$. that will take part in the review process is se. 'cte all this TTs are shown in the zone marked $v$, th a i in Figure 5 , where the exact location o , TT 1 sb wn with a marker in the map that allows he on to see the type of the terrain of every tower. All ie TT parameters (id, location, last review date, ground sistivity, resistance, $\mathrm{kr}$ and frequented type) are shown in a dialog when the user interacts with the map by clicking on the marker (marked with 'd' in Figure 5).

The module marked in Figure 5 with the letter ' $b$ ' offers the possibility of establishing the configuration that will be taken into account when estimating the values for a specified existing or new TT.

The execution of the module marked with 'a' (by clicking on the 'Sample' button) shows the user the results of the execution of the sampling algorithm and the negotiation process. Results are shown in different ways: (i) the towers to review are detailed in a map with markers, (ii) grouped by their type and resistivity values, and (iii) listed individually. In addition, information about previous reviews and the reduction percentage achieved by the sampling algorithm for the selected input data are shown. An output example can be seen in Figure 6, where a $67.23 \%$ reduction is achieved s 39 TT are proposed to be reviewed from a total of 119 selected TT. Internally, the system ensures that the proposed TT have the highest influence on future revision processes if reviewed; however, the negotiation result is not shown to final users as it is not relevant to them.

Furthermore, the execution of the estimation functionality to predict the values of a future TT, can help in determining the best TT model to use, the ground resistivity in the specified location and the resistance of the TT at that point, so the best location to be placed can be found. This functionality can be used as shown in Figure 7. The step marked with the number 1 consists of establishing the set of TTs to be considered. If it is not possible to establish a set of TTs, the whole database is used. In the second step (number 2 in Figure 7), users can specify different parameters related to the neighbors, such as the maximum number of neighbors to consider and the longest distance to be considered as a neighbor, in addition to other parameters such as temperature or humidity. The new TT location is also established by introducing the coordinates or by interacting with the map. The result of the prediction can be found in the 3rd step of the Figure 7. In this case, the predicted values for the specified TT (colored in red in the image) and the influence in the estimation of its neighbors (neighbors are colored in blue) is represented with lines colored in a scale from green to red, 


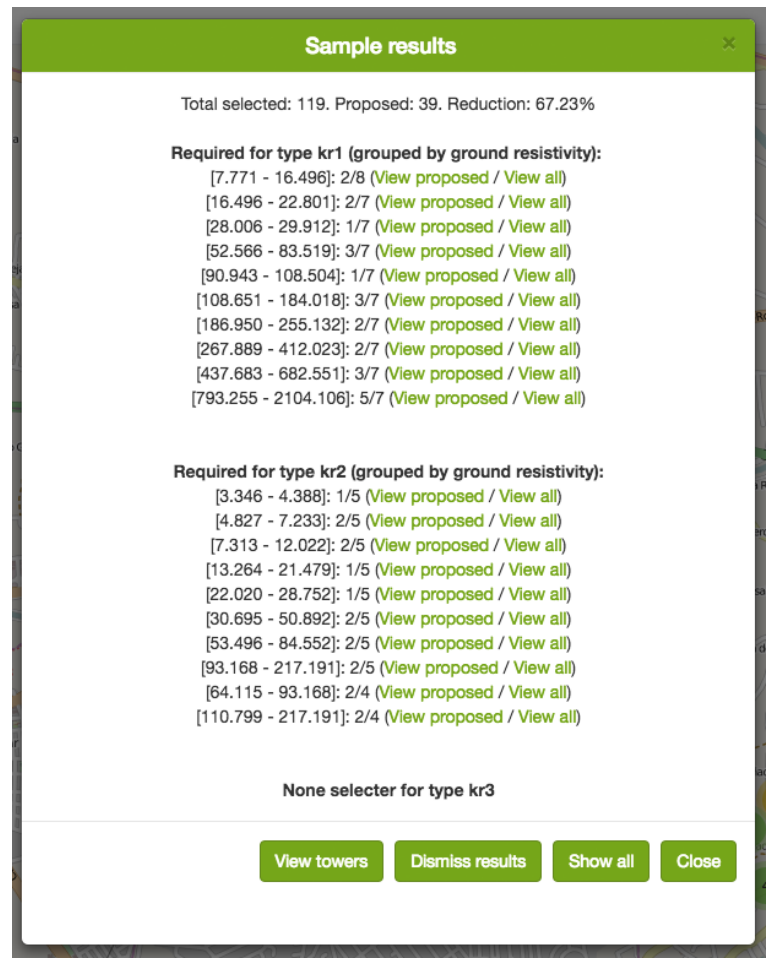

Fig. 6. Sampling of a set of TT.

where green represents the highest influenc or all estimation of that neighbor and red represe ts th lowe influence.

To evaluate the system, different $v_{c}$ 'les $f$ the ground resistivity, resistance of the ' 1 "s an he $K_{r}$ of the TTs were measured, in ac 'itu to e....rnal values influencing the values, such as aperature or humidity (on a scale of high, medium - low). More specifically, 3,000 real reviews of TTs from different areas of Spain were carried out by following the same process.

With these real data, different estimations were carried out in order to validate the proposed prediction procedure of the system for both the ground resistivity and the TT resistance. Estimations were performed over a subset of 100 TTs (40 TTs belong to group 1, 40 TTs belong to group 2, and 20 TTs belong to group 3 , which is the least common) extracted from the 3,000 TTs with real values. These 100 TTs were not inserted in the database. Different configurations were evaluated for the estimation of every TT to evaluate how parameters such as the maximum distance or the number of neighbors influence the result. The average distance for the neighbors was also extracted for the evaluation. With this procedure, the system results are shown in table 2 .
Table 2

Accuracy of the estimation.

\begin{tabular}{|c|c|c|c|}
\hline TT & Neighbors & Average distance (km) & Accuracy (\%) \\
\hline \multirow{3}{*}{$K_{r} \mathbf{1}$} & 10 & 3.56 & 98.65 \\
\cline { 2 - 4 } & 50 & 8.77 & 97.2 \\
\cline { 2 - 4 } & 100 & 19.37 & 95.4 \\
\hline \multirow{3}{*}{$K_{r} \mathbf{2}$} & 10 & 4.3 & 98.47 \\
\cline { 2 - 4 } & 50 & 12.23 & 96.83 \\
\cline { 2 - 4 } & 100 & 25.34 & 95.01 \\
\hline \multirow{3}{*}{$K_{r} \mathbf{3}$} & 10 & 8.31 & 97.2 \\
\cline { 2 - 4 } & 50 & 20.32 & 93.77 \\
\hline \multirow{3}{*}{ All } & 100 & 52.5 & 94 \\
\cline { 2 - 4 } & 10 & $\mathbf{1} 81$ & 98.29 \\
\cline { 2 - 4 } & 100 & $\mathbf{1 2 . 4 0}$ & 96.37 \\
\hline
\end{tabular}

The accuracy was c lcu ted according to the success of the predi in. If the real value belongs to the range $c$ valu s predicted by the system (the predicted value - the error), the estimation is considere $\rightarrow$ successful. The accuracy does not appear to have be . Infl enced by the model of the TT, but it has a direu relationship with the average distance of the neighbors. It seems clear that the lower the number of considered neighbors, the lower the average distance. Nonetheless, the system (equation (3)) prioritizes closest neighbors over those further away, so the accuracy still shows high values although the average distance is increased.

When evaluating the percentage of reduction that the system achieves for the set of selected TTs, results were analyzed by taking two parameters into account: (i) the number of selected TTs, and (ii) the average distance between all the selected TTs. Ground resistivity values are directly affected by the distance since the further the distance is, the higher is the possibility to have different types of terrain with different resistivity values; therefore, closest TTs are expected to be placed in more similar terrains. Similarly, the more TTs are selected, the more possibilities to find other TTs with values similar to those in the selected set, which involves better results. The reduction percentage that the system achieved by following the described procedure is shown in table 6. The data set is composed of approximately 80,000 TTs in Spain with both real data (gathered with the 3,000 reviews that have been previously mentioned) and estimated data (based on historical inventory data).

The most significant reduction is achieved as a greater number of TTs is selected for review. However, a lower level reduction is achieved when TTs are far 


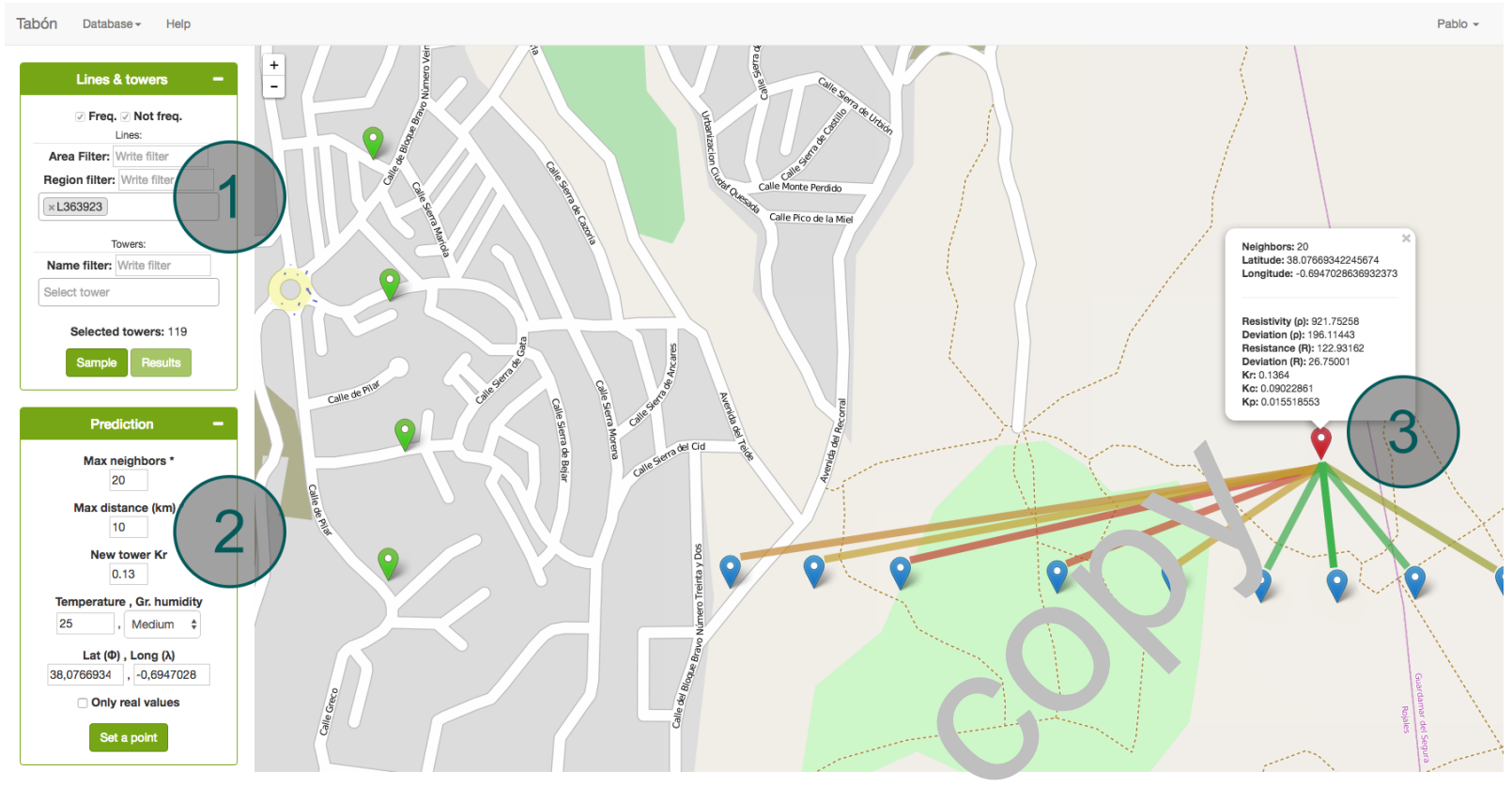

Fig. 7. Prediction of a ew Tm

Table 3

Percentage of reduction when sampling.

\begin{tabular}{|c|c|c|c|}
\hline TT & Distance (km) & Proposed TT & Reduction (\%) \\
\hline 100 & 50 & 27 & 73 \\
\hline 200 & 50 & 35 & 8 \\
\hline 500 & 50 & 64 & 7.2 \\
\hline 800 & 50 & 83 & 89.6. \\
\hline 800 & 300 & 154 & 75 \\
\hline
\end{tabular}

apart, even when the number or $\mathrm{T} / 1 \mathrm{~s}$ high. Obviously, the farther TTs are located fron other TTs, the more different their values are. When reviewing TTs in real life, the reduction only makes sense when working on a specific area or power line, so this problem is never going to be faced.

As it takes too long to evaluate the revision process with real deterioration data (Spanish law set that TTs review period 4 years for frequented TTs), different deterioration simulations were performed to allow the evaluation of the negotiation process that determines which TTs must be reviewed from each group. A simulation example is presented below, where the system proposed 28 TTs from a set of 100 as a result of executing the sampling algorithm. The TTs to be reviewed were proposed by following three different criteria: first, (i) the TTs to be reviewed were proposed randomly (RandX) by selecting 28 TTs from the original set of 100. Second, (ii) the system used a deter- ministıc method (DetX) where always the TTs with the 'owest $T P$ are proposed to be reviewed, in this case, 28 T Ts. This condition should be the optimal method if the TP of other TTs are not influenced when other TTs are reviewed. In this case, the $T P$ value is influenced if other TT is reviewed because they can have similar behaviors if the values of their parameters are similar, as mentioned before. Finally, the third method (iii) is the proposed negotiation system (RevX). A graphic with the TP growth for the three methods is shown in Figure 8 . In the described example, the highest reachable mean value is 96.4. This is because in the best of circumstances, there would be 28 reviewed TTs whose $T P$ value is equal to 100 , and the other 72 TTs could have a value up to 95 , which is the maximum percentage guaranteed by the system as determined by $z$ in the equation (5).

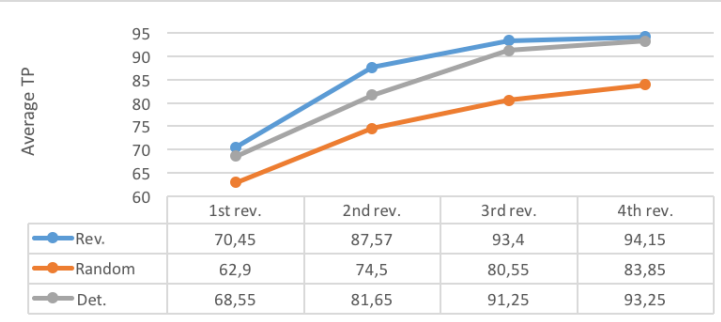

Fig. 8. $T P$ evolution in the 4 stages. 
The Figure 8 shows that the proposed negotiation system (in blue) reaches the highest $T P$ value during the first revision, and the second revision stage reaches a value even higher than the fourth stage of the random system (orange). In addition, the value of the fourth stage reaches an average value of 94.15 , which guarantees a really high reliability on the values of the parameters of all the selected TTs.

The dispersion of the $T P$ values that these three methods provided is shown in the following figures ( 9 , 10 , and 11). In the random system, the plot scatter is higher. On the other hand, when solved using both the negotiation system and the deterministic method, values improve in conjunction in every review stage. In the fourth stage we can see that there are no $T P$ values lower than 80, the exact opposite of the random method where values lower than 30 can be found. Nevertheless, when using the deterministic method, the proposed negotiation system presents the highest average $T P$ value (AvgNeg4 in Figure 11) and lower $T P$ values on the fourth stage. The negotiation mechanism would therefore appear to provide the best results, which involves a higher reduction on the maintenance costs when using this method.

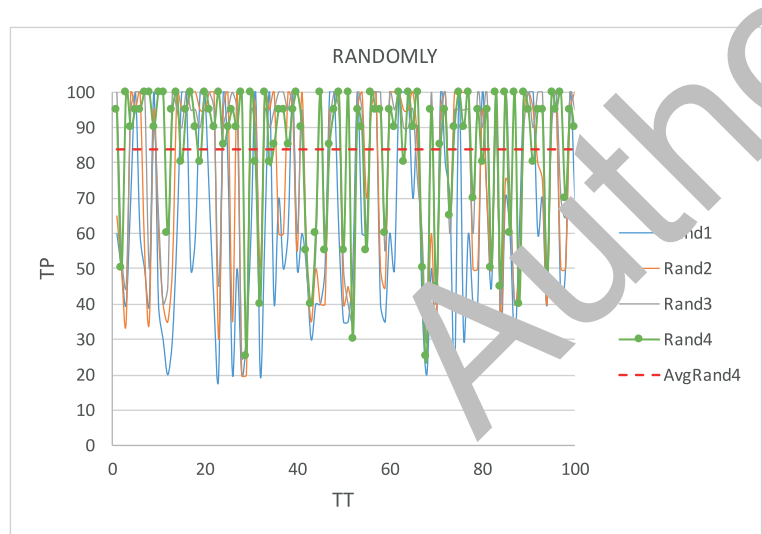

Fig. 9. Values for the set of 100 TT with the random method in the four simulated stages.

In order to determine the statistical significance for differences and observe the evolution between the three methods, Figure 12 presents a box-plot. It is useful for analyzing the evolution of $T P$ according to the different methods. Graphically, it is clear that the $T P$ average is higher for every method when increasing the interaction number; nevertheless, the dispersion of the random method (Rand) decreases less than other methods and it presents outliers in the third iteration.

The deterministic (Det) method presents higher dispersion than the negotiation method (Rev) proposed

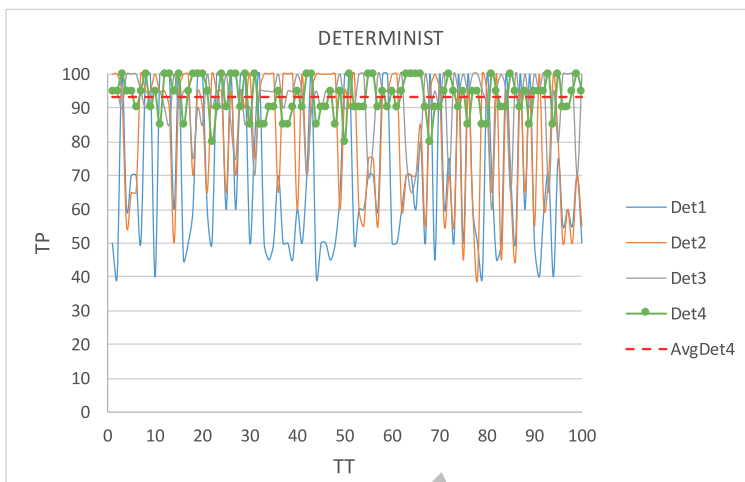

Fig. 10. Values for the set of $100 \mathrm{~T}_{\mathrm{T}} \mathrm{it}_{\mathrm{t}}$ the deterministic method in the four simulated stages.

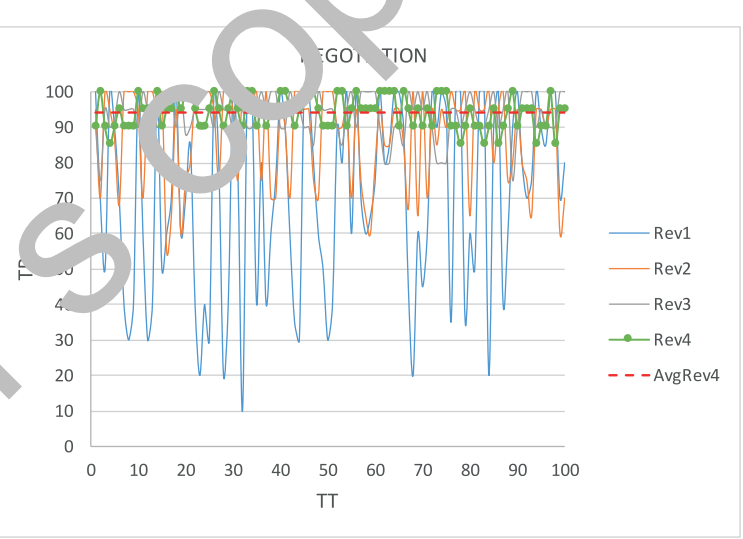

Fig. 11. Values for the set of 100 TT with the negotiation method in the four simulated stages.

from the first iteration. The proposed method is the one with the highest improvement and graphically shows the best $T P$ in the fourth iteration by analyzing the dispersion. The average of the proposed method is higher in the first iteration and average values tend to be equal because the remaining towers are reviewed in the following iterations.

In order to analyze the significance of the results obtained, a Mann Whitney statistical test is used. H1 indicates that the row has a $T P$ value higher than the column $T P$ value. Results are shown in table 4, where significant differences are highlighted in bold. When analyzing the values, it is clear that the $T P$ value for Rev2 is better than the value for Det2 and Rand2. In stage 3 , the results are more similar between Det 3 and Rev3, and also in stage 4 between Det 4 and Rev4. It could not therefore be argued that there are statistical difference even though the average for the negotiation method is higher than the deterministic average. 
Table 4

$P$ value obtained after applying Mann Whitney. H1 means that the row has a $T P$ value higher than the column $T P$ value

\begin{tabular}{|c|c|c|c|c|c|c|c|c|c|c|c|c|}
\hline & Det1 & Rand1 & Rev1 & Det2 & Rand2 & Rev2 & Det3 & Rand3 & Rev3 & Det4 & Rand4 & Rev1 \\
\hline Det1 & & $\mathbf{0 , 0 3 3}$ & 0,723 & 1,000 & 0,800 & 1,000 & 1,000 & 0,996 & 1,000 & 1,000 & 1,000 & 1,000 \\
\hline Rand1 & 0,967 & & 0,968 & 1,000 & 0,987 & 1,000 & 1,000 & 1,000 & 1,000 & 1,000 & 1,000 & 1,000 \\
\hline Rev1 & 0,278 & 0,032 & & 0,995 & 0,830 & 1,000 & 1,000 & 0,994 & 1,000 & 1,000 & 0,999 & 1,000 \\
\hline Det2 & 0,000 & 0,000 & 0,005 & & 0,053 & 0,960 & 0,993 & 0,387 & 0,997 & 0,997 & 0,691 & 0,998 \\
\hline Rand2 & 0,201 & 0,013 & 0,171 & 0,947 & & 0,995 & 0,998 & 0,858 & 0,999 & 0,999 & 0,941 & 0,999 \\
\hline Rev2 & 0,000 & 0,000 & 0,000 & 0,040 & $\mathbf{0 , 0}$ & & 0,893 & 0,097 & 0,971 & 0,963 & 0,269 & 0,984 \\
\hline Det3 & 0,000 & 0,000 & 0,000 & 0,007 & 0,002 & 0,108 & & 0,026 & 0,687 & 0,581 & 0,048 & 0,793 \\
\hline Rand3 & 0,004 & 0,000 & 0,006 & 0,614 & 0,143 & 0,903 & 0,975 & & 0,984 & 0,972 & 0,646 & 0,990 \\
\hline Rev3 & 0,000 & 0,000 & 0,000 & 0,003 & 0,001 & $\mathbf{0 , 0 3 0}$ & 0,313 & 0,016 & & 0345 & 0,019 & 0,664 \\
\hline Det4 & 0,000 & 0,000 & 0,000 & 0,003 & 0,001 & 0,038 & 0,420 & 0,028 & 0,65 & & $\mathbf{7 , 0 2 8}$ & 0,818 \\
\hline Rand4 & 0,000 & 0,000 & 0,001 & 0,310 & 0,059 & 0,732 & 0,953 & 0,355 & 0, , & 0,72 & 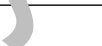 & 0,992 \\
\hline Rev4 & 0,000 & 0,000 & 0,000 & 0,002 & 0,001 & 0,016 & 0,208 & 0,011 & 07 & $\bar{n} . \overline{83}$ & $\mathbf{0 , 0 0 8}$ & \\
\hline
\end{tabular}

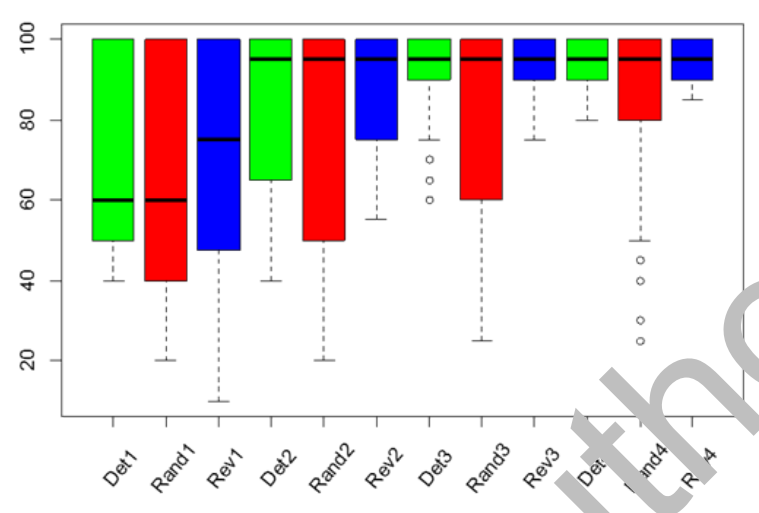

Fig. 12. Box-plot with the $T P$ value for the threc lifere it methods in the four stages.

\section{Conclusions and Fu ure Work}

This work proposes a model of artificial intelligence that can predict the values of different parameters of TT with a high accuracy, and sample the number of TTs to review. With this system, it is possible to reduce the maintenance cost of the power transport infrastructure. By using VO and AT it is possible to propose a system capable of reducing the number of measurements in TT, although there are factors that cannot be controlled, such as undetectable environmental changes that alter soil resistivity.

The system presented is able to decide autonomously which TTs must be reviewed. The reduction amount of the initial sample depends directly on parameters such as the distance between them, the similarity of the resistance, soil resistivity and $K_{r}$ values.

Sampling is useful when correct data are available. If predicted values or previous values are significantly different fr on tho sbtained when measured, more TTs are lik $1 \mathrm{y}$ to e wrong. In this case, all the initial sample shoulu ue reviewed in order to solve additional err r.r.

ine fu ure work for this project is influenced by the need to increase the real case database in order to eval'late the system with more real data to get better results which are not dependent on the ground resistivity estimation.

In addition, the negotiation benefits will be shown with the following revision stages, allowing the system to be evaluated without being simulated.

\section{Acknowledgements}

This work has been supported by the European Commission H2020 MSCARISE-2014: Marie

Skłodowska-Curie project DREAM-GO Enabling Demand Response for short and real-time Efficient And Market Based Smart Grid Operation - An intelligent and real-time simulation approach ref 641794 .

The research of Pablo Chamoso has been financed by the Regional Ministry of Education in Castille and León and the European Social Fund (Operational Programme 2014-2020 for Castille and León, EDU/310/2015 BOCYL).

\section{References}

[1] A. Badri, A. N. Niazi, and S. M. Hoseini. Long term preventive generation maintenance scheduling with network constraints. Energy Procedia, 14:1889-1895, 2012. 
[2] H. de Faria, J. G. S. Costa, and J. L. M. Olivas. A review of monitoring methods for predictive maintenance of electric power transformers based on dissolved gas analysis. Renewable and Sustainable Energy Reviews, 46:201-209, 2015.

[3] F. De la Prieta, S. Rodríguez, J. Bajo, and J. M. Corchado. A multiagent system for resource distribution into a cloud computing environment. In International Conference on Practical Applications of Agents and Multi-Agent Systems, pages 37-48. Springer, 2013.

[4] P. Do, A. Voisin, E. Levrat, and B. Iung. A proactive conditionbased maintenance strategy with both perfect and imperfect maintenance actions. Reliability Engineering \& System Safety, 133:22-32, 2015

[5] España. Real decreto-ley 1955/2000, de 1 de diciembre, por el que se regulan las actividades de transporte, distribución, comercialización, suministro y procedimientos de autorización de instalaciones de energía eléctrica. boletín oficial del estado, 27 de diciembre de 2000, núm. 310. http://www.boe.es/buscar/pdf/2000/ BOE-A-2000-24019-consolidado . pdf. Accessed: 2016-0630.

[6] R. E. Española. Gestor de la red y transportista. http://www.ree.es/es/actividades/ gestor-de-la-red-y-transportista, 2015. Accessed: 2016-06-30.

[7] Y. Feng, W. Wu, B. Zhang, and J. Gao. Transmission line maintenance scheduling considering both randomness and fuzziness. Journal of Uncertain Systems, 5(4):243-256, 2011.

[8] A. Fipa. Fipa acl message structure specification. For dation for Intelligent Physical Agents, http. "w' w. " $a$. org/specs/fipa00061/SC00061G. html (30.6. 2ר04),. 102.

[9] S. Heras, V. Botti, and V. Julián. On a computauc 71 ars nentation framework for agent societies. In Inl natu Workshop on Argumentation in Multi-Agen rystem nages 123140. Springer, 2010.

[10] K. Ji, X. Rui, L. Li, A. Leblon and - McClure. A novel ice-shedding model for overhead wer line conductors with the consideration of adhesive/cohe ve forces. Computers \& Structures, 157:153-164, 2015.

[11] K. Krishnanand, P. Dash, and M. Naeem. Detection, classification, and location of faults in power transmission lines. International Journal of Electrical Power \& Energy Systems, 67:7686, 2015.

[12] R. Murugan and R. Ramasamy. Failure analysis of power transformer for effective maintenance planning in electric utilities. Engineering Failure Analysis, 55:182-192, 2015.

[13] S. Ossowski, C. Sierra, and V. Botti. Agreement technologies: A computing perspective. In Agreement Technologies, pages 3-16. Springer, 2013.

[14] A. Sánchez, G. Villarrubia, C. Zato, S. Rodríguez, and P. Chamoso. A gateway protocol based on fipa-acl for the new agent platform pangea. In Trends in Practical Applications of Agents and Multiagent Systems, pages 41-51. Springer, 2013.

[15] S. A. Taher and I. Sadeghkhani. Estimation of magnitude and time duration of temporary overvoltages using ann in transmission lines during power system restoration. Simulation Modelling Practice and Theory, 18(6):787-805, 2010.
[16] A. J. Trappey, C. V. Trappey, L. Ma, and J. C. Chang. Intelligent engineering asset management system for power transformer maintenance decision supports under various operating conditions. Computers \& Industrial Engineering, 84:3-11, 2015.

[17] F. Wenner. A method for measuring earth resistivity. Journal of the Franklin Institute, 180(3):373-375, 1915.

[18] A. Zarnani, P. Musilek, X. Shi, X. Ke, H. He, and R. Greiner. Learning to predict ice accretion on electric power lines. Engineering Applications of Artificial Intelligence, 25(3):609-617, 2012.

[19] C. Zato, G. Villarrubia, A. Sánchez, I. Barri, E. Rubión, A. Fernández, C. Rebate, J. A. Cabo, T. Álamos, J. Sanz, et al. Pangea-platform for automatic construction of organizations of intelligent agents. In Distribu $d$ Computing and Artificial Intelligence, pages 229-239. ^rin $r$, 2012.

[20] D. Zhou, H. Zhang, an' r. 'Veng. novel prognostic model of performance degra ${ }^{1}$ tion to nd for ower machinery maintenance. Energy, 7Q.74८ $746 \quad 014$. 